\title{
Correction to: Key Role of Disulfide Bridges in the Antimicrobial Activity of Beta-Defensin from Olive Flounder
}

\author{
Yunqi Ma ${ }^{1}$ - So-Sun Kim ${ }^{1}$. Chang-Hyun Maeng ${ }^{1}$ - David Nahm-Joon Kim ${ }^{1}$. Chang-Joo Lee ${ }^{1}$ - Bo-Hye Nam ${ }^{2}$. \\ Youg-Ok Kim ${ }^{2} \cdot$ Cheul-Min $\mathrm{An}^{2} \cdot$ Jang-Su Park ${ }^{1}$ (1)
}

Published online: 13 April 2019

(c) Springer Nature B.V. 2019

\section{Correction to: \\ International Journal of Peptide Research and Therapeutics https://doi.org/10.1007/s10989-019-09837-z}

The original version of this article unfortunately contained a typo in the author name and in Acknowledgement section.

The author name should be Youg-Ok Kim instead it was published incorrectly as Yong-Ok Kim.

In Acknowledgement section, the university name should be National Institute of Fisheries Science instead it was published incorrectly as National Fisheries Research and Development Institute. The correct complete funding information is as follows:
The original article can be found online at https://doi.org/10.1007/ s10989-019-09837-z.

\section{Jang-Su Park}

jaspark@ pusan.ac.kr

Bo-Hye Nam

nambohye@korea.kr

1 Department of Chemistry and Chemistry Institute of Functional Materials, Pusan National University, Busan 609-735, Republic of Korea

2 Biotechnology Research Division, Aquaculture Industry Department, National Fisheries Research and Development Institute, 408-1 Sirang-ri, Gijang-eup, Gijang-gun, Busan 619-902, Republic of Korea
This research was supported by grants from the Marine Biotechnology Program (Grant No. 20170305; Development of Biomedical materials based on marine proteins) Funded by the Korean Ministry of Oceans and Fisheries, and from the National Institute of Fisheries Science (R2019016).

The original article has been corrected. 